\title{
Non-secreting adrenal myelolipoma in a middle-aged male patient manifesting with sudden onset of severe lower back pain
}

\author{
Carlo Introini $^{1}$, Fabio Campodonico ${ }^{1}$, Marco Ennas ${ }^{1}$, Antonia Di Domenico ${ }^{1}$, Luca Foppiani ${ }^{2}$ \\ ${ }^{1}$ Urology, ${ }^{2}$ Internal Medicine, Galliera Hospital, Genoa, Italy.
}

\begin{abstract}
Summary Adrenal myelolipoma (AML) is a rare benign tumor, usually non-functioning and asymptomatic until it reaches large size. AML is mostly detected incidentally by imaging and is composed of adipose tissue and hematopoietic elements. Only symptomatic tumor needs surgical excision. We report the case of a large non-functioning adrenal tumor discovered by means of combined imaging techniques in a middle-aged male patient who complained the sudden onset of severe lower back pain; successful laparoscopic removal was performed, and AML was diagnosed at histopathology.
\end{abstract}

KEY WORDS: Adrenal myelolipoma; Tumor size; Laparoscopy.

Submitted 2 March 2020; Accepted 15 March 2020

\section{INTRODUCTION}

Adrenal myelolipoma (AML) is a benign tumor composed of variable amounts of mature adipose tissue and bone marrow (hematopoietic) elements. AML has an overall prevalence at autopsy of $0.0 .8-0.2 \%$, affects patients in their fifth to seventh decades of life, and is usually asymptomatic and non-functioning (1).

With the widespread use of ultrasonography (US), computed tomography (CT) and magnetic resonance imaging (MRI), the finding of AML has reached up to $7-15 \%$ of adrenal incidentalomas $(2,3)$. Albeit mostly asymptomatic, huge AML may cause flank pain and abdominal discomfort/pain by causing pressure of surrounding structures and may present with rupture, haemorrhage, or hemorrhagic shock $(1,3)$. In patients with AML three distinct types of endocrine dysfunctions have been described: hormone secreting AML, AML occurring in patients with congenital adrenal hyperplasia (CAH) and AML occurring in association with a secreting adrenal co-lesion. The rare secreting AMLs display cortisol or aldosterone secretion; nevertheless, a few reports of catecholamine and androgen secretion have been described $(1,3)$.

We report the case of a large non-functioning AML which was discovered in a middle-aged male patient presenting with sudden lower back pain.

\section{Case report}

A 47-year-old man was admitted to the emergency department for severe lower right back pain and vomiting. Physical examination was unremarkable, and blood pressure and blood tests were normal. Abdomen US showed a large $9 \times 6 \mathrm{~cm}$ hyperechoic curved lesion with regular edge localized in the right suprarenal region. Contrast-enhanced CT confirmed a large $9 \mathrm{~cm}$ elliptical heterogeneous neoplasm with regular margins localized in the right adrenal gland, which featured a hypodense part interspersed with more dense component (Figure la), and proved suspicious for pheochromocytoma.

The patient was admitted to the urology ward. In view of surgical removal of the adrenal mass, hormonal array was carried out on both plasma (ACTH, cortisol, 17-hydroxyprogesterone, DHEAS, upright renin and aldosterone) and urine (24-h free cortisol, 24-h metanephrines and normetanephrines); all values proved unremarkable. Laparoscopic adrenalectomy was performed by transperitoneal approach. In lumbotomic position four trocars were placed by configuring a diamond draw. This technique offers a wide visualization of the operative field ensuring minimal morbidity. The $12-\mathrm{mm}$ port was inserted at the lateral border of the rectus abdominis muscle just above the level of the umbilicus to accommodate the camera. Two subcostal 5-mm ports were placed; one in the midclavicular line and the other in the lateral border of the rectus abdominis muscle.

The third 5-mm subcostal trocar was inserted in the anterior axillary line. The upper pole of the right kidney was mobilised and the huge adrenal lesion was found (Figure 1b, c). Once the mobilization of the lesion was completed, the tumor was removed en-bloc by enlarging the pararectal incision. On gross examination, a $9 \times 6 \mathrm{~cm}$ reddish yellow colored and friable mass was observed. Histology showed normally shaped mature adipocytes mixed with hematopoietic cells and surrounded by a capsule; these features were compatible with the diagnosis of myelolipoma. Postoperative course was uneventful and the patient was discharged after 5 days.

\section{Discussion}

AML is discovered incidentally in 2-5\% of population during imaging analysis performed for other reasons. Tumor size varies from several millimeters to more than 40 centimeters $(1,3)$. The fat component of AML is postulated to be derived by the mesenchymal stem cells harbouring in the vessel wall of stromal fat of adrenal cortex. The mature adipocytes become inflammatory and stimulate adrenal cortex tissue to release substances (likely granulocyte colony stimulating factor) that recruit 
Figure 1.

Contrast-enhanced computed tomography scan demonstrating a huge inhomogeneous right adrenal mass containing amount of low attenuation tissue of fatty density (a, arrow), a large well capsulated round mass was found at surgery (b), the adrenal gland was entirely dissected along the capsule (c).
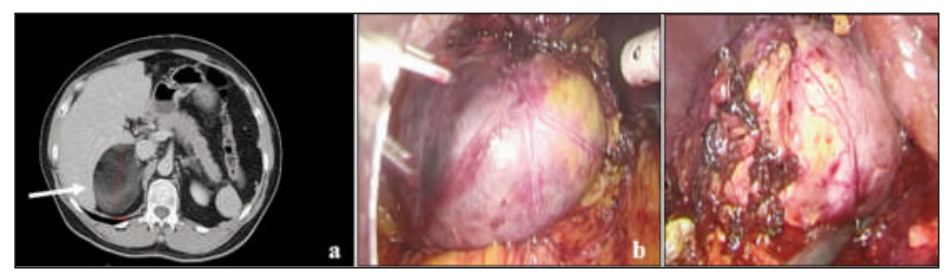

circulating haematopoietic progenitors to anchor and differentiate (4). The diagnosis of AML is usually made by means of various imaging techniques (US, CT and MRI). CT is considered the most sensitive way to detect these lesions, since AMLs show low attenuation values (-10 to -30 Hounsfield units) and mixed density. Although not diagnostic, the presence of fat density within the mass is a very useful feature to characterize a lesion as a myelolipoma (5).

A recent review on 420 cases of AML ascertained that the average size was $10 \mathrm{~cm}$; CAH was present in $10 \%$ of patients, whereas cortisol or aldosterone secretion was found in $7.5 \%$ of cases (1). In our patient the size of the adrenal mass was in line with literature data and no hormonal secretion was ascertained.

Asymptomatic AMLs which measure less than $4 \mathrm{~cm}$ do not require further investigation (hormonal evaluation or follow-up imaging). Surgical treatment becomes necessary in those rare tumours which are functional or increase in size or become symptomatic. AMLs larger than $6 \mathrm{~cm}$ are at high risk of spontaneous rupture with haemorrhage leading to acute abdomen and shock and should be removed $(1,3)$.

In our patient, a large $(9 \mathrm{~cm})$ AML became symptomatic (severe back pain) and required surgery. However, the CT features of the right adrenal mass were initially deemed suggestive of pheochromocytoma: this tumor, in fact, may show homogeneous or variable enhancement as was found in our patient (5), and this may puzzle the diagnosis. Hormonal work-up was therefore carried-out. The normality of 24-h urinary metanephrine and normetanephrine levels and blood pressure prompted us to exclude a pheochromocytoma. In addition, normal 17-hydroxyprogesterone levels ruled out CAH; finally, normal ACTH and cortisol levels together with the absence of Cushingoid's features likely excluded a cortisol-secreting AML. The Clinical Practice Guidelines of European Society of Endocrinology do not recommend in AML the endocrine/metabolic work-up deemed mandatory in other adrenal incidentalomas (2).

However endocrine dysfunctions are reported in 7-10\% of $\operatorname{AML}(1,3)$. In our opinion, given the increasing number of AML reported as secreting hormones or occurring in association with either CAH or adrenal collision tumors (adrenal carcinoma, cortisol-secreting adenoma, aldosterone-secreting adenoma), endocrine work-up seems advisable at least in those patients with hypertension, diabetes, or bilateral AML. From a urological point of view is consolidated that the organ most suitable for the laparoscopic approach is the adrenal gland; since it is deeply located in the retroperitoneum a large incision is required in case of open surgery. The right adrenalectomy, specifically in case of tumor diameter larger than $5 \mathrm{~cm}$, can be more challenging due to the proximity with the inferior vena cava and the duodenum (6).

A recent study compared the results in two groups of patients who underwent single-site transumbilical laparoendoscopic adrenalectomy (LA) for adrenal tumors or standard multi-port LA. No difference in terms of operative time, blood loss, analgesic requirement, and hospital stay was found; however in the first group $85 \%$ of patients did not require drainage compared to $25 \%$ of patients in the second group, and resumed normal diet earlier (7).

\section{Conclusions}

AML is a rare, benign, and usually asymptomatic tumor of adrenal gland. Hormonal work-up seems worthwhile at least in those patients at high metabolic risk or who are referred to surgery. In particular, rare catecholaminesecreting AMLs must be ruled out before surgery in order to prevent life-threatening hypertensive crisis during intraoperative tumor manipulation.

The AMLs which are symptomatic owing to hormonal secretion or more frequently to mass effect should undergo surgical excision. Laparoscopic adrenalectomy is nowadays the preferred and safe surgical option. Informed consent was obtained from the patient.

\section{References}

1. Decmann A, Perge P, Tóth M, Igaz P. Adrenal myelolipoma: a comprehensive review. Endocrine. 2018; 59:7-15.

2. Fassnacht M, Arlt W, Bancos I, et al. Management of adrenal incidentalomas: European Society of Endocrinology Clinical Practice Guideline in collaboration with the European Network for the Study of Adrenal Tumors. Eur J Endocrinol. 2016; 175:G1-G34.

3. Shenoy VG, Thota A, Shankar R, Desai MG. Adrenal myelolipoma: Controversies in its management. Indian J Urol. 2015; 31:94-101.

4. Feng C, Jiang H, Ding Q, Wen H. Adrenal myelolipoma: a mingle of progenitor cells? Med Hypotheses. 2013; 80:819-22.

5. Wale DJ, Wong KK, Viglianti BL, et al. Contemporary imaging of incidentally discovered adrenal masses. Biomed Pharmacother. 2017; 87:256-262.

6. Gunseren KO, Cicek MC, Vuruskan H, et al. Challenging risk factors for right and left laparoscopic adrenalectomy: a single centre experience with 272 case. Int Braz J Urol. 2019; 45:747-753.

7. Carvalho JA, Nunes PT, Antunes H, et al. Transumbilical laparoendoscopic single-site adrenalectomy: a feasible and safe alternative to standard laparoscopy. Arch Ital Urol Androl. 2019; 91:1-4.

\section{Correspondence}

Carlo Introini, MD (Corresponding Author) - carlo.introini@galliera.it

Fabio Campodonico, MD - fabio.campodonico@galliera.it

Marco Ennas, MD - marco.ennas@galliera.it

Antonia Di Domenico, MD - antonia.didomenico@galliera.it

Urology, Galliera Hospital, Genova (Italy)

Luca Foppiani, MD, PhD (Corresponding Author) - luca.foppiani@galliera.it Internal Medicine, Galliera Hospital

Mura delle Cappuccine 14, 16128 Genova (Italy) 